\title{
Modelagem dinâmica e cenários urbanos de demanda de água: simulações em Campina Grande (PB)
}

\author{
Dynamic modeling and urban water demand scenarios: \\ simulations in Campina Grande-PB
}

\section{Laís Marques de Oliveira Almino' ${ }^{1}$, lana Alexandra Alves Rufino ${ }^{1 *}$ (])}

\begin{abstract}
RESUMO
Este trabalho propõe estimativas de demanda de água para cenários futuros de uso e ocupação do solo e de verticalização utilizando análises espaciais e modelagem dinâmica com base em autômatos celulares para uma fração urbana do município de Campina Grande (PB). Dados reais de uso e ocupação do solo e de verticalização dos anos de 2011 e2018 são utilizados para identificar o processo de mudança nesses bairros. Um conjunto de variáveis estáticas e dinâmicas relacionadas é selecionado para subsidiar a parametrização do modelo e simular as mudanças no uso e ocupação do solo e na verticalização, ocorridas no período. Após a validação das simulações para o ano de 2018 com base nos dados observados, novos cenários futuros são propostos para os anos de 2040, 2070 e 2100, identificando, assim, uma tendência de ocupação com algumas características específicas, como a substituição de áreas residenciais de um ou dois pavimentos por novos empreendimentos verticais, seja residencial, empresarial ou de serviços. Dado o aumento de ocupação urbana, as demandas de água capazes de atender à população também aumentam, aumento este que se confirmou neste trabalho. Entre os anos 2018 e 2100, essa tendência de aumento foi estimada em 300\%. Uma comparação de cenários futuros considerando usos convencionais e racionais de água (utilizando mecanismos poupadores) também é apresentada.
\end{abstract}

Palavras-chave: modelagem dinâmica; autômatos celulares; uso e ocupação do solo; verticalização; demanda de água.

\begin{abstract}
This work estimates urban water demands for future land-use scenarios using spatial analysis and dynamic modeling based on cellular automata for an urban area of the city of Campina Grande, Paraiba State, Brazil. Ground truth for two dates (2011 and 2018) is used to identify the process of land-use change in these neighborhoods. Some static and dynamic variables are defined in order to support the model parameterization and to simulate the changes occurred in the period. After the validation of those simulations for 2018, based on the observed data, new future scenarios are proposed for the years 2040 , 2070, and 2100, thus identifying a tendency of occupation with some specific characteristics such as the replacement of residential areas new buildings with multiple floors, whether residential, business, or services. Due to the increase in urban occupation, the water demands for attending to the population also increase. Between 2018 and 2100, this upward trend is estimated at 300\%. A comparison of future scenarios considering conventional and rational water uses (using saving mechanisms) is also simulated.
\end{abstract}

Keywords: dynamic modeling; cellular automato; soil use and occupation; verticalization; water demand.

\section{INTRODUÇÃO}

A intensificação do processo de urbanização e o rápido crescimento das cidades, em âmbito mundial, foram uns dos fatos mais marcantes do século XX. A urbanização no Brasil intensificou-se com a modernização da agricultura e o advento da industrialização. A concentração das indústrias transformou as grandes cidades em polos de desenvolvimento cultural, educacional e tecnológico, e, enquanto provedoras de empregos, as indústrias foram capazes de atrair significativo contingente populacional. Como consequência, o adensamento populacional e o desenvolvimento das atividades econômicas incorporaram uma nova organização na configuração espacial dessas cidades, bem como novas estruturas sociais e econômicas (ROSSETTI; ALMEIDA; PINTO, 2013).

口-

'Programa de Pós-Graduação em Engenharia Civil e Ambiental, Universidade Federal de Campina Grande - Campina Grande (PB), Brasil!

*Autora correspondente: iana.alexandra@ufcg.edu.br

Conflitos de interesse: os autores declaram não haver conflito de interesses.

Financiamento: Coordenação de Aperfeiçoamento de Pessoal de Nivel Superior (Capes), Conselho Nacional de Desenvolvimento Científico e Tecnológico (CNPq) (PQ N 313323/2017-8 e PDE N. 205565/2018-2) e Projeto Segurança Hídrica, Alimentar e Energética na Bacia Estendida do Rio São Francisco (MCTI/CNPq N. 19/2017). Recebido: 16/01/2019 - Aceito: 12/10/2019 - Reg. ABES: 20190015 
O acelerado crescimento populacional urbano tornou-se um desafio para os estudiosos do meio urbano. A busca pela compreensão das mudanças que se processam no espaço e no tempo confere importância à obtenção de possíveis previsões, concretizando-se assim em ferramentas de auxílio ao planejamento e ordenamento territorial (TRENTIN; FREITAS, 2010).

$\mathrm{Na}$ opinião de Ferrari e Lapolli (2000), esse desafio se torna ainda maior quando o crescimento das cidades está associado à verticalização, pois, neste caso, os problemas de infraestrutura são mais complexos, dado o adensamento populacional ocasionar sobrecarga na infraestrutura já existente.

Tendo em vista todos esses processos de expansão urbana e verticalização acelerados, surge a necessidade de entender melhor a dinâmica do espaço urbano e, com isso, planejar melhor as cidades para futuras adaptações e/ou enfrentamento de eventos extremos, tornando-as mais resilientes. Nesse sentido, o objetivo deste trabalho é estimar a demanda de água em cenários futuros de uso e ocupação do solo e de verticalização, por meio de análises espaciais e da parametrização e calibração de um modelo de simulações de mudanças, destinado a gerar simulações futuras para os anos 2040, 2070 e 2100 em dois bairros no município de Campina Grande (PB).

\section{MODELAGEM DINÂMICA ESPACIAL}

De acordo com Roy e Snickars (1996), os conceitos de autômatos celulares foram inicialmente expostos por John Von Newmann na década de 1960. Em 1970, John Conway deu grande popularidade a esses conceitos, quando apresentou o "Jogo da Vida", (do inglês: The Game of Life), em que demonstrou que regras muito simples, quando aplicadas repetidamente sobre estados aleatórios, produzem resultados semelhantes à forma como certos sistemas evoluem no mundo real (GARDNER, 1970).

Meirelles, Camara e Almeida (2007) afirmam que a modelagem dinâmica espacial se baseia na modelagem de ecossistemas com extensões para acomodar a heterogeneidade espacial e os processos humanos de tomada de decisão. A principal característica da modelagem dinâmica espacial é a facilidade com que é utilizada na descrição da dimensão espacial, assim como o fato de descrever quantitativamente um fenômeno e prever sua evolução, integrando suas escalas temporal e espacial.

Algumas abordagens de modelagem em "células" (como modelagem baseada em agentes) tornaram-se populares para fenômenos como propagação de doenças (JOHANSSON et al., 2012), crime (MALLESON; BIRKIN, 2011) e uso da terra (MATTHEWS et al., 2007). Mais recentemente, Jat, Choudhary e Saxena (2017) apresentaram possibilidades de integração de ferramentas do sensoriamento remoto, o Sistema de Informação Geográfica (SIG), e de um modelo baseado em autômatos celulares (AC), o SLEUTH (do inglês: Slope, Land cover, Exclusion, Urbanization, Transportation and Hillshade), de uma área urbana de maior complexidade dada a sua grande heterogeneidade.

Esses modelos espaciais dinâmicos são utilizados principalmente para a modelagem espaço-temporal de processos, sobretudo os físicos e os de planejamento urbano-regional, servindo para explicar a ocorrência de um determinado fenômeno, seu padrão espacial e sua evolução ao longo do tempo (ALMEIDA; CAMARA; MONTEIRO, 2007; MEIRELLES; CAMARA; ALMEIDA, 2007; SUAREZ; SOARES-FILHO, 2013).

Para Rossetti, Almeida e Pinto (2013), a associação das tecnologias de sensoriamento remoto e geoprocessamento a modelos dinâmicos espaciais utilizados em análise urbana permitem avaliar de forma quantitativa a estruturação e a dinâmica do espaço urbano, proporcionando uma melhor visualização da realidade urbana e dos elementos responsáveis pelas suas transformações espaço-temporais.

A modelagem espacial dinâmica é usada, entre outras finalidades, para entender as alterações do uso e cobertura da terra, possibilitando, ainda, a realização de simulações de mudanças na paisagem como potencial ferramenta para antecipar prováveis tendências passíveis de ocorrerem em uma região (ALMEIDA, 2003; DELANEZE, 2011; GONÇALVES; CENTENO; CANDEIAS, 2011; MAEDA et al., 2011; DIAS; WALDE, 2013).

Segundo Mas et al. (2014), as mudanças no uso e cobertura do solo têm chamado bastante atenção de cientistas e tomadores de decisões. Nesse sentido, alguns estudos foram realizados com o intuito de analisar e modelar espaços urbanos, utilizando as ferramentas disponíveis na modelagem dinâmica espacial. Por exemplo, modelagem da tendência histórica do crescimento urbano de Karaj - Irã (SAKIEH et al., 2015), crescimento urbano com mudanças associadas ao uso da terra e desagregação do solo no Nordeste da China (GONG et al., 2015) e modelagem dos padrões de expansão urbana da região metropolitana de São Paulo (MASSABKI et al., 2017), apesar de nenhum deles ter relacionado a modelagem espacial do crescimento urbano com a análise de disponibilidade hídrica.

\section{CARACTERIZAÇÃO HÍDRICA DA ÁREA DE ESTUDO}

A gestão da demanda urbana de água torna-se ainda mais necessária em centros urbanos de regiões áridas/semiáridas de países em desenvolvimento (caso de grande parte do Nordeste brasileiro), onde as condições climáticas, aliadas a sistemas de abastecimento obsoletos e à inadequação ou à ausência de um planejamento urbano sensível aos recursos hídricos, determinam o surgimento de graves problemas de abastecimento de água, dificultando o atendimento das demandas quantitativas e qualitativas da população (FIGUERES, 2005; RÊGO et al., 2013).

Inserido nessa realidade, destaca-se o caso da cidade de Campina Grande, segunda maior cidade paraibana e importante polo educacional, industrial e tecnológico do estado. Essa cidade tem aspectos peculiares para esta pesquisa, pois enfrenta crises hídricas recorrentes com riscos elevados de um desabastecimento da água potável (GUEDES; RIBEIRO; VIEIRA, 2014; BARROS; RUFINO; MIRANDA, 2016).

Rêgo, Albuquerque e Ribeiro (2000), durante a crise que teve seu início em 1997 e seu fim em 1999, afirmavam que ela se deu quando uma prolongada seca afetou a região Nordeste do Brasil. O açude público Epitácio Pessoa, responsável pelo abastecimento de Campina Grande e outras cidades do compartimento da Borborema, atingiu o mais baixo nível de sua história até então ( $15 \%$ da capacidade máxima de armazenamento), submetendo mais de $500 \mathrm{mil}$ pessoas a severo racionamento de água, por um período superior a dois anos.

Na opinião de Rêgo, Albuquerque e Ribeiro (2000) e Galvão et al. (2001), nesse período foi possível constatar a ausência de efetiva gestão de recursos hídricos. A cidade continuou expandindo-se, e, em 2012, deu-se início a um novo período de estiagem no semiárido brasileiro, provocando uma nova crise no sistema de abastecimento de água da cidade de Campina Grande (RÊGO; GALVÃO; ALBUQUERQUE, 2012; RÊGO et al., 2013), dessa vez, com impactos ainda maiores.

Em 2017, o reservatório Epitácio Pessoa (Boqueirão) sofreu risco de colapso, chegando a ficar com apenas $2,9 \%$ da sua capacidade total (AESA, 
2019). Toda a situação de risco de colapso vivenciada em 2017 já era esperada desde o início da estiagem em 2012, caso não ocorressem períodos chuvosos. Sendo assim, observando que realmente não estavam acontecendo eventos de chuva, para evitar esse colapso e garantir o prolongamento do abastecimento de água da cidade, instaurou-se em dezembro de 2014 um severo racionamento de água que se perpetuou até a chegada do eixo leste da transposição do rio São Francisco, que passou a garantir um pouco mais de segurança hídrica ao município. O crescimento de Campina Grande sob essas condições climáticas específicas faz com que a população perceba claramente o impacto ambiental causado pela urbanização crescente (escassez hídrica causando desabastecimento de água potável e submetendo a população a racionamentos frequentes). Essas situações de crises frequentes despertam um comportamento de resiliência e racionalidade que tem estimulado mudanças na construção civil, como o uso de sistemas de captação de água de chuva (no microclima urbano há eventos concentrados de chuva que não contribuem para o reservatório em questão) e de mecanismos poupadores nos edifícios e residências de uma forma geral.

Campina Grande está localizada no agreste do estado da Paraíba, a $7^{\circ} 13^{\prime} 50^{\prime \prime}$ de latitude Sul e a $35^{\circ} 52^{\prime} 52^{\prime \prime}$ de longitude Oeste. O município apresenta uma área territorial de $593,03 \mathrm{~km}^{2}$, sendo cerca de $91,00 \mathrm{~km}^{2}$ os limites da zona urbana, e uma população de 407.472 habitantes, dos quais $95,33 \%$ compreendem a população urbana e 4,67\% a população rural (IBGE, 2018a). A área de estudo (Figura 1) escolhida compreende os bairros Catolé e Sandra Cavalcante.

Nos bairros em estudo estão localizados importantes equipamentos urbanos de grande porte, tais quais: terminal interurbano de passageiros (rodoviária), shopping center, grandes escolas, clubes, parque, entre outros, configurando duas regiões importantes da cidade. Em razão desses equipamentos urbanos, os bairros cresceram muito em população e, de acordo com dados do último censo (IBGE, 2010), apresentam alta densidade populacional urbana, $6.782 \mathrm{hab} . / \mathrm{km}^{2}$ e $4.959 \mathrm{hab} . / \mathrm{km}^{2}$, respectivamente. Essa densidade já é certamente maior pelo grande adensamento ocorrido após a coleta de dados censitários oficiais em 2010.

Araújo (2012) fez uma simulação de consumo de água para diferentes cenários e alertou para a necessidade de um planejamento urbano que considere a capacidade de abastecimento humano e a infraestrutura de expansão da rede. Barros, Rufino e Miranda (2016) constataram, ao analisarem dados do censo 2000 e 2010, um aumento na verticalização em média de 335 para 476 apartamentos $/ \mathrm{km}^{2}$ no bairro Catolé e de 135 para 295 apartamentos $/ \mathrm{km}^{2}$ no bairro Sandra Cavalcante.

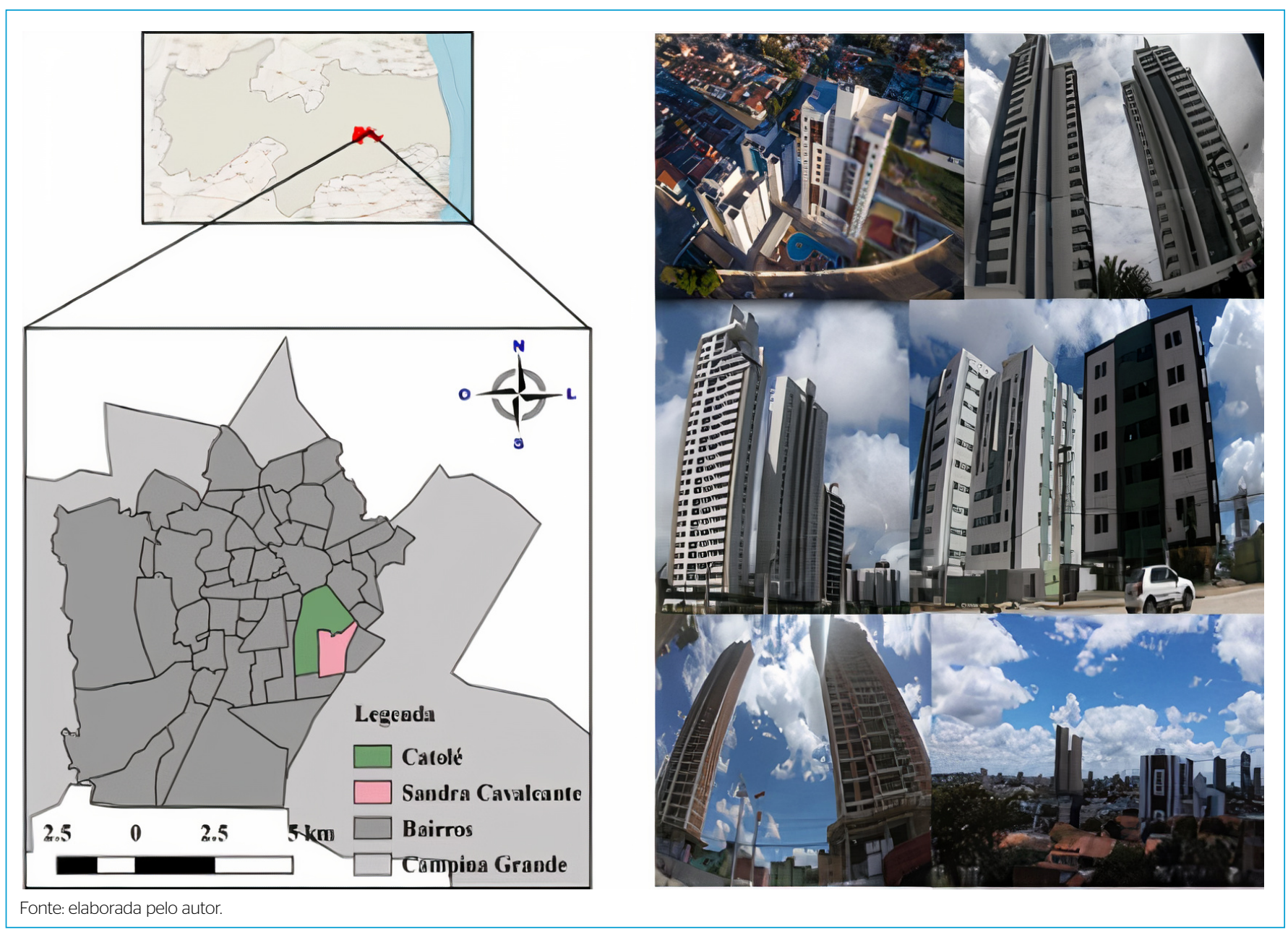

Figura 1 - (A) Localização da área de estudo. (B) Imagens demonstrando o grau de urbanização da área. 


\section{METODOLOGIA}

\section{Modelagem espacial: cenários passados, presentes e futuros}

Neste trabalho, consideraram-se os seguintes cenários de uso e ocupação do solo para compor os dados de entrada da modelagem dinâmica:

- cenário I: referente ao ano de 2011;

- cenário II: referente ao ano de 2018 (observado em campo e simulado por meio de um software baseado em AC);

- cenários III, IV e V: cenários simulados com base na matriz de transição (matriz de mudanças do uso do solo e verticalização) ocorrida entre os cenários I e II, referentes aos anos de 2040, 2070 e 2100.

A escolha dos cenários deve-se em parte à disponibilidade de dados passados (caso do cenário I), dados espacializados da área de estudo presentes no estudo realizado por Araújo (2012). No cenário II, todas as informações foram coletadas em campo ao longo de vários meses de visitas in loco e observações durante o ano de 2018. Os cenários futuros de longo prazo (cenários III, IV e V), mesmo tendo um período de mudança curto (inferior a 10 anos de mudança) para ser aplicado em sua simulação, foram escolhidos com o intuito de utilizar os resultados gerados neste artigo em simulações e cruzamentos de dados com estudos de mudança climática que usualmente utilizam períodos de 30 anos para gerar modelos. A Figura 2 é uma representação esquemática das etapas metodológicas deste estudo.

O modelo baseado em AC utilizado foi o software Dinâmica EGO (acrônimo para Environment for Geoprocessing Objects ou ambiente para geoprocessamento de objetos), desenvolvido pela equipe do Centro de Sensoriamento Remoto da Universidade Federal de Minas Gerais (CSR/UFMG). Na edição e preparação dos cenários de entrada, utilizam-se planilhas eletrônicas, além do software QGIS

Os mapas de uso e ocupação do solo e de verticalização dos bairros Catolé e Sandra Cavalcante - de 2011e de 2018 - podem ser visualizados nas Figuras 3 e 4. Os cálculos das áreas (em km² e em percentuais) para os cenários I (2011) e II (2018) em ambiente SIG permitiram uma quantificação das tendências de aumento ou diminuição de determinada classe de uso e ocupação do solo ou de verticalização.

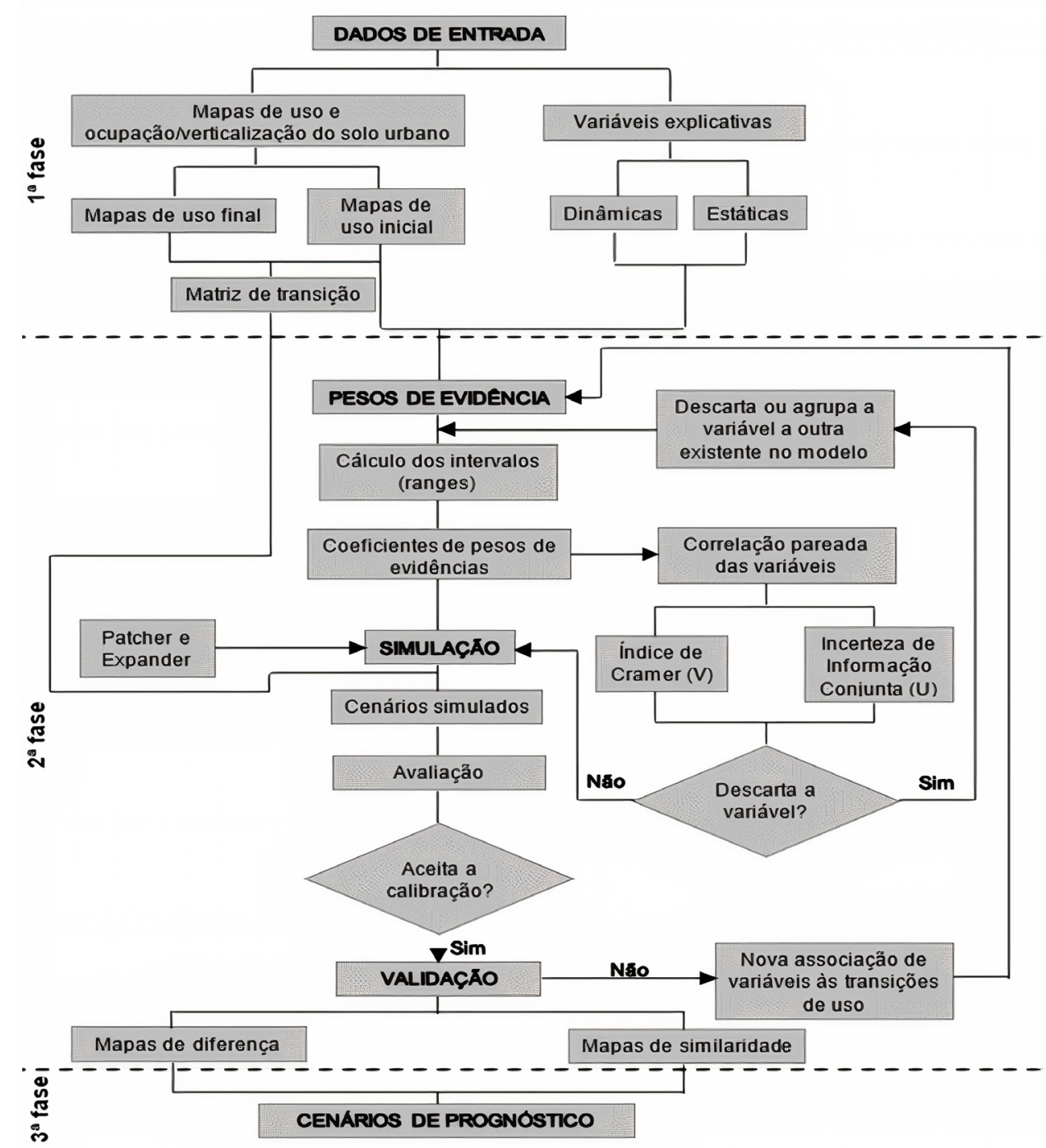

Fonte: adaptada de Trentin (2008)

Figura 2 - Síntese das etapas de modelagem dinâmica espacial no software de simulação Dinâmica EGO. 


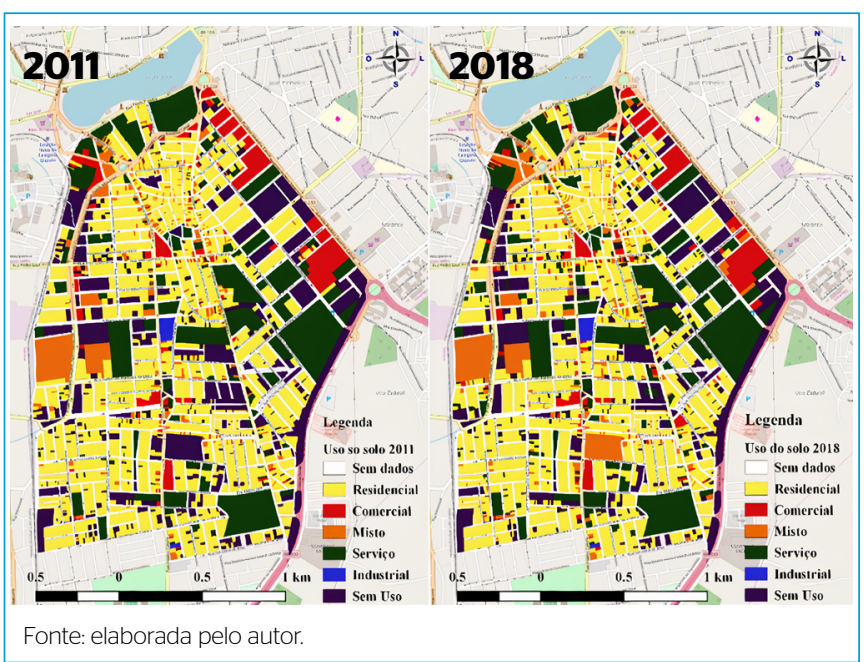

Figura 3 - Representações do uso e ocupação do solo nos bairros Catolé e Sandra Cavalcante (2011 e 2018).

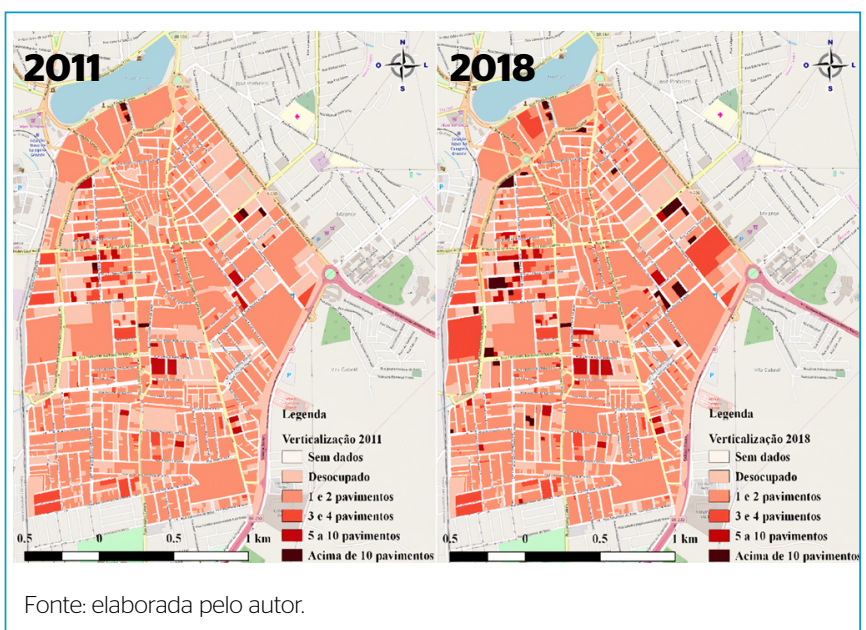

Figura 4 - Representações da verticalização dos bairros Catolé e Sandra Cavalcante (2011 e 2018).

\section{Variáveis estáticas e variáveis dinâmicas}

A acurácia e a eficiência desse modelo dependem diretamente da qualidade dos dados de entrada. Só com base em cenários passados e presentes com detalhamento compatível torna-se possível a elaboração de cenários futuros consistentes. Além disso, para "treinar" o modelo de acordo com uma tendência de uso e ocupação observada, faz-se necessária a seleção de variáveis explicativas das mudanças observadas. As variáveis que descrevem as modificações da área de estudo nesse ambiente podem ser de dois tipos: variáveis dinâmicas e variáveis estáticas. As variáveis dinâmicas recebem esse nome porque são atualizadas ao longo da simulação, enquanto isso não ocorre com as variáveis estáticas. Essas variáveis foram selecionadas conforme a Tabela 1.

No caso do estudo, foram feitas duas simulações distintas. Para ambas as simulações, utilizaram-se todas as variáveis estáticas. A variável que diferenciava uma simulação da outra era a dinâmica, para a simulação de uso e ocupação do solo, a variável dinâmica utilizada foi “distância a áreas já utilizadas". Já para a simulação de verticalização, a variável dinâmica utilizada foi "distância a áreas já verticalizadas".
Tabela 1 - Variáveis utilizadas na modelagem.

\begin{tabular}{l|r} 
Variáveis estáticas & Variáveis dinâmicas \\
Distância às instituições de ensino particular & $\begin{array}{r}\text { Distância a áreas já utilizadas } \\
\text { (1a simulação) }\end{array}$ \\
\cline { 1 - 1 } Distância aos principais shoppings da cidade & $\begin{array}{r}\text { Distância a áreas já verticalizadas } \\
\text { (2a simulação) }\end{array}$ \\
\hline Distância aos vias arteriais & \\
\hline Distância às áreas de lazer & \\
\hline
\end{tabular}

Fonte: elaborada pelo autor.

\section{Calibração e validação do modelo}

A calibração do modelo consiste em quatro etapas: cálculo das matrizes de transição, cálculo dos coeficientes dos pesos de evidência, análise da correlação dos mapas e ajuste e execução do modelo de simulação. A matriz de transição é responsável por fornecer percentual de mudanças de uma classe para a outra ao algoritmo de simulação. Essa informação é obtida por meio da tabulação cruzada do mapa de classes inicial e final. Nesse segmento, são gerados dois tipos de matriz, de passos simples e de passos múltiplos (ROSSETTI; ALMEIDA; PINTO, 2013). Com base nos mapas inicial e final, calcularam-se taxas de transição em um passo simples, ou seja, do período completo (sete anos) e em passos múltiplos (anualizadas).

O método dos pesos de evidência é aplicado no Dinâmica EGO para produzir um mapa de probabilidades de transição, que representa as áreas mais favoráveis para mudança. Segundo Gonçalves, Sousa Junior e Almeida (2007), o mapa de probabilidades de transição tem papel fundamental no entendimento do processo de mudança. Para categorizar as variáveis, são calculados intervalos nos mapas de distâncias, de forma semelhante a um fatiamento.

Isso consiste em um método bayesiano, no qual o efeito de uma variável espacial em uma transição é calculado independentemente de uma solução combinada. Os pesos das evidências representam cada influência em uma variável na probabilidade espacial de uma transição $i \rightarrow j$ e são calculados conforme as Equações 1 e 2:

$O\{\mathrm{D} \mid \mathrm{B}\}=\frac{P\{\mathrm{D} \mid \mathrm{B}\}}{P\{\overline{\mathrm{D}} \mid \mathrm{B}\}}$

$\log \{\mathrm{D} \mid \mathrm{B}\}=\log \{D\}+W^{+}$

Em que:

$W^{+}=$o peso da evidência para a ocorrência do evento $\mathrm{D}$, dado um padrão espacial B. A probabilidade a posteriori de uma transição $\mathrm{i} \rightarrow \mathrm{j}$, dado um conjunto de dados espaciais (B, C, D, .., N), é expresso conforme a Equação 3:

$P\{i \rightarrow j \mid B \cap C \cap D \ldots \cap N\}=\frac{e^{\Sigma W_{N}^{+}}}{1+e^{\Sigma W_{N}^{+}}}$

Em que:

B, C, D e N = os valores das variáveis espaciais contidas nos mapas e representadas por seus pesos $W_{N}^{+}$.

A única suposição necessária ao método dos pesos de evidência é que os mapas de entrada devem ser espacialmente independentes, em que os pares de variáveis estáticas e dinâmicas contidas nos mapas devem ser independentes 
espacialmente. Para avaliar essa independência, realizou-se a análise da correlação dos mapas, ou seja, essa análise valida ou não os pesos de evidência calculados para serem utilizados na simulação. Para testar essa independência, todos os pares de variáveis estáticas com dinâmicas e estáticas com estáticas são analisados. Na opinião de Bonham-Carter (1994), um grupo de medidas pode ser aplicado para estimar essa suposição, como o teste de Cramer (V) e o joint information uncertainty (incerteza de informação conjunta - JIU). Esses índices operam com valores reais e percentuais, respectivamente, e avaliam o grau de dependência espacial entre pares de variáveis. Conforme Bonham-Carter (1994), quanto mais próximos do valor 1 esses índices estiverem, maior a dependência espacial entre os pares de variáveis consideradas. Além disso, pares de variáveis com correlação acima de 0,5 (50\%) devem ser desprezados ou combinados em uma terceira variável que substituirá o par correlacionado no modelo.

A parametrização do modelo também inclui o ajuste e a execução do modelo de simulação. O Dinâmica EGO usa como regra local de AC um mecanismo de transição composto de dois functores complementares de transição: Patcher e Expander. A função Patcher destina-se a gerar novas manchas, por meio de um mecanismo de constituição de sementes. A função Expander responde pela expansão de manchas previamente existentes de uma determinada classe (SOARES-FILHO; CERQUEIRA; PENNACHIN, 2002).

Além da definição da proporção entre Patcher e Expander, é necessário definir o tamanho médio e a variância da área de mudança, além do índice de isometria. Todos esses parâmetros (Tabelas 2 e 3) são inseridos na fase "Patcher e Expander" da simulação na Figura 2.

Tabela 2 - Parâmetros utilizados no ajuste e na execução do modelo de simulação de uso e ocupação do solo.

\begin{tabular}{l|c|c|c|c}
\multicolumn{5}{|c}{ Uso do solo } \\
\hline Tipo & $\begin{array}{c}\text { Tamanho } \\
\text { médio (ha) }\end{array}$ & $\begin{array}{c}\text { Variância } \\
\text { (ha) }\end{array}$ & Isometria & $\begin{array}{c}\text { Patcher/ } \\
\text { Expander }\end{array}$ \\
\hline Residencial & 1,64 & 2,40 & 1,5 & 0,6 \\
\hline Comercial & 0,80 & 3,00 & 1,5 & 0,6 \\
\hline Misto & 0,40 & 2,56 & 1,5 & 0,6 \\
\hline Serviço & 1,84 & 4,44 & 1,5 & 0,6 \\
\hline Industrial & 1,72 & 2,84 & 1,5 & 0,6 \\
\hline Sem Uso & 0,88 & 2,80 & 1,5 & 0,6 \\
\hline
\end{tabular}

Fonte: elaborada pelo autor.

Tabela 3 - Parâmetros utilizados no ajuste e na execução do modelo de simulação de verticalização.

\begin{tabular}{l|c|c|c|c}
\multicolumn{5}{c}{ Verticalização } \\
Classes & $\begin{array}{c}\text { Tamanho } \\
\text { médio (ha) }\end{array}$ & $\begin{array}{c}\text { Variância } \\
\text { (ha) }\end{array}$ & Isometria & $\begin{array}{c}\text { Patcher/ } \\
\text { Expander }\end{array}$ \\
\hline Desocupado & 0,88 & 2,80 & 1,5 & 0,6 \\
\hline 1 e 2 pavimentos & 2,72 & 4,12 & 1,5 & 0,6 \\
\hline 2 e 3 pavimentos & 0,60 & 2,04 & 1,5 & 0,6 \\
\hline 5 a 10 pavimentos & 1,00 & 1,80 & 1,5 & 0,6 \\
\hline $\begin{array}{l}\text { Acima de 10 } \\
\text { pavimentos }\end{array}$ & 0,64 & 0,96 & 1,5 & 0,6 \\
\hline
\end{tabular}

Fonte: elaborada pelo autor.
Esses parâmetros são usados para padronizar tamanhos das manchas que surgem ou se expandem dos existentes à medida que as transformações ocorrem. O tamanho médio, a variância e a isometria da área de mudança foram obtidos por meio do método de tentativa e erro. $\mathrm{O}$ índice de isometria varia de $0 \mathrm{a} 2$, as manchas assumem uma forma mais isométrica à medida que esse número aumenta. $\mathrm{O}$ grau de fragmentação das manchas é inversamente proporcional ao valor do índice (ALMEIDA et al., 2008).

Os valores dos parâmetros utilizados na fase "Patcher e Expander" são ajustados até se obter uma calibração aceitável, ou seja, obter um mapa simulado que represente de maneira fiel o mapa real. Isso foi feito por meio de uma análise de diferença (comparação) no software QGIS entre os mapas de 2018 (real e simulado).

Após a geração de um mapa 2018 simulado, com base no mapa inicial 2011, que representasse a situação real vista em campo, comprovando a obtenção dos parâmetros satisfatórios, partiu-se para a etapa de validação. Com as quatro etapas da calibração concluídas, o desempenho do modelo foi validado pela similaridade fuzzy. O índice de similaridade fuzzy empregado neste trabalho foi criado pelo CSR/UFMG e representa uma adaptação do índice de similaridade fuzzy criado por Hagen (2003). No caso particular deste trabalho, a comparação foi feita entre dois mapas-diferenças, resultantes, de um lado, da subtração entre o mapa final real (2018 real) e o mapa inicial (2011) e, de outro lado, entre o mapa final simulado (2018 simulado) e o mapa inicial (2011), conforme implementado na plataforma Dinâmica EGO. Adotou-se uma função de decaimento exponencial com tamanho de janela 11x11 e uma função de decaimento constante, calculada com os seguintes tamanhos de janelas: 1x1, 3×3, 5x5, 7x7, 9x9 e 11x11. Os tamanhos das janelas são grades de pixels. No caso da janela 11x11, a validação por comparação é feita por meio de grades contendo 11 pixels verticais e 11 pixels horizontais, em que, neste estudo, cada pixel corresponde a uma área de $25 \mathrm{~m}^{2}(5 \mathrm{~m} \mathrm{x} 5 \mathrm{~m}$ ). Com o modelo validado, três cenários futuros de uso e ocupação do solo e de verticalização foram gerados, de acordo com o escopo da modelagem. A Figura 5 é uma representação esquemática desse processo.

\section{Simulação de cenários futuros}

Após a validação do modelo, os mesmos parâmetros de ajuste utilizados para calibrar o modelo foram utilizados para simular os cenários futuros de uso e

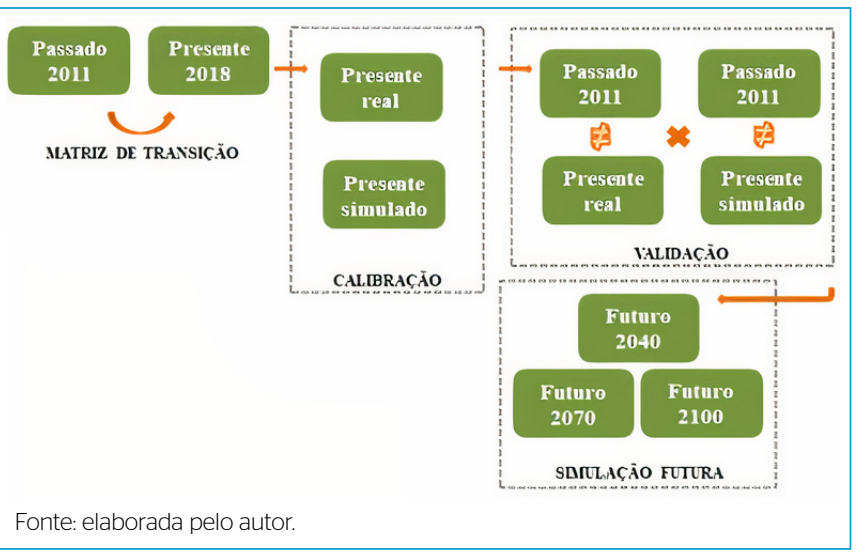

Figura 5 - Representação esquemática da calibração do modelo. 
ocupação do solo e de verticalização, mudando apenas o número de interações do modelo. Nas simulações para os mapas dos anos 2040, 2070 e 2100, empregaram-se 22, 52 e 82 interações, respectivamente (referentes aos intervalos de anos para cada cenário). Interações feitas agora com base no mapa de 2018 e não mais do de 2011.

Para melhor representação das mudanças que ocorreram em curto prazo (de 2011 a 2018) em anos futuros de longo prazo (2040, 2070 e 2100), preferiu-se utilizar as taxas de transição em passos múltiplos (anualizadas) para as simulações do modelo.

\section{Estimativas e validação do consumo de água}

Inicialmente, a estimativa do consumo de água foi realizada para cada lote, para o cenário de uso e ocupação do solo correspondente ao ano de 2018. Para realizar essa estimativa, utilizaram-se dados coletados em campo (tipo de ocupação, número de pavimentos), no site do Instituto Brasileiro de Geografia e Estatística (IBGE) (população dos bairros), no site da Relação Anual de Informações Sociais (RAIS) (IBGE, 2018b) (número de funcionários por estabelecimento comercial), na Companhia de Água e Esgoto da Paraíba (Cagepa) e no Sistema Nacional de Informações sobre Saneamento (SNIS) (consumo per capita). Todos esses dados foram essenciais para utilizar metodologias encontradas na literatura, como a Norma da Companhia de Saneamento Básico do Estado de São Paulo (SABESP, 2012) e Tsutyia (2006), já que existem metodologias para o cálculo do consumo residencial e comercial.

O cálculo do consumo residencial estimado, detalhado em Tsutyia (2006), foi feito por meio da taxa de consumo normal e racional por habitante equivalente a 151,3 L/hab.dia disponível no SNIS (2018) (correspondente ao ano de 2013 antes do período de seca) e a 97,1 L/hab.dia disponibilizado pela Cagepa (correspondente ao ano 2016 - durante o período de seca), respectivamente. Já o cálculo do consumo comercial estimado foi feito por meio da metodologia detalhada pela Norma da Sabesp (2012), utilizando o número de funcionários por estabelecimento comercial, que, por sua vez, foi obtido pelo site da RAIS. Esse dado de número de funcionários também foi utilizado para consumo industrial e serviço.

Para os demais cenários simulados (2040, 2070 e 2100), não foi possível realizar esse nível de detalhamento (por lote) na estimativa da demanda de água, dada a natureza dos dados ser matricial após a simulação. No entanto, para esses cenários, estimou-se a demanda de água pela utilização de uma taxa de consumo de água "por pixel", da quantidade de pixel para cada tipo de uso do solo e do fator de verticalização (demanda de água para os cenários futuros simulados $(1 /$ dia $)=$ taxa de consumo de água por pixel $\mathrm{x}$ número de pixel de cada tipo de uso do solo $\mathrm{x}$ fator de verticalização). A Figura 6 mostra a síntese das etapas realizadas para estimar a demanda de água futura.

A tendência do aumento da demanda de água é observada para dois cenários: de consumo racional e de consumo convencional. São estimadas, em ambos os cenários, as diferenças entre essas demandas de água com (CMP) e sem (SMP) a utilização de mecanismos poupadores em edifícios residenciais, como: torneira (com arejador), chuveiro, bacia sanitária (de acionamento duplo), reuso de água (cinzas) e medidores individuais.

Segundo Barros, Rufino e Miranda (2016), a utilização desses mecanismos poupadores em edifícios residenciais gera uma economia de $10,42 \%$ no consumo

É importante ressaltar que, após a etapa da estimativa do consumo de água para o cenário II (2018), os consumos estimados foram validados. Essa validação foi necessária para se ter a confiabilidade de aplicar esses dados para os cenários futuros de demanda de água (2040, 2070 e 2100). Os dados de consumo medidos foram disponibilizados pela Cagepa (2018). Disponibilizaram-se os dados de consumo micromedido de água mensais da área de estudo (dos bairros Catolé e Sandra Cavalcante).

A metodologia utilizada nessa etapa de validação foi a de comparação direta entre os consumos de água estimados calculados e os consumos de água medidos

Agrupamento do consumo estimado de água para cada tipo de uso do solo no cenário II (2018)

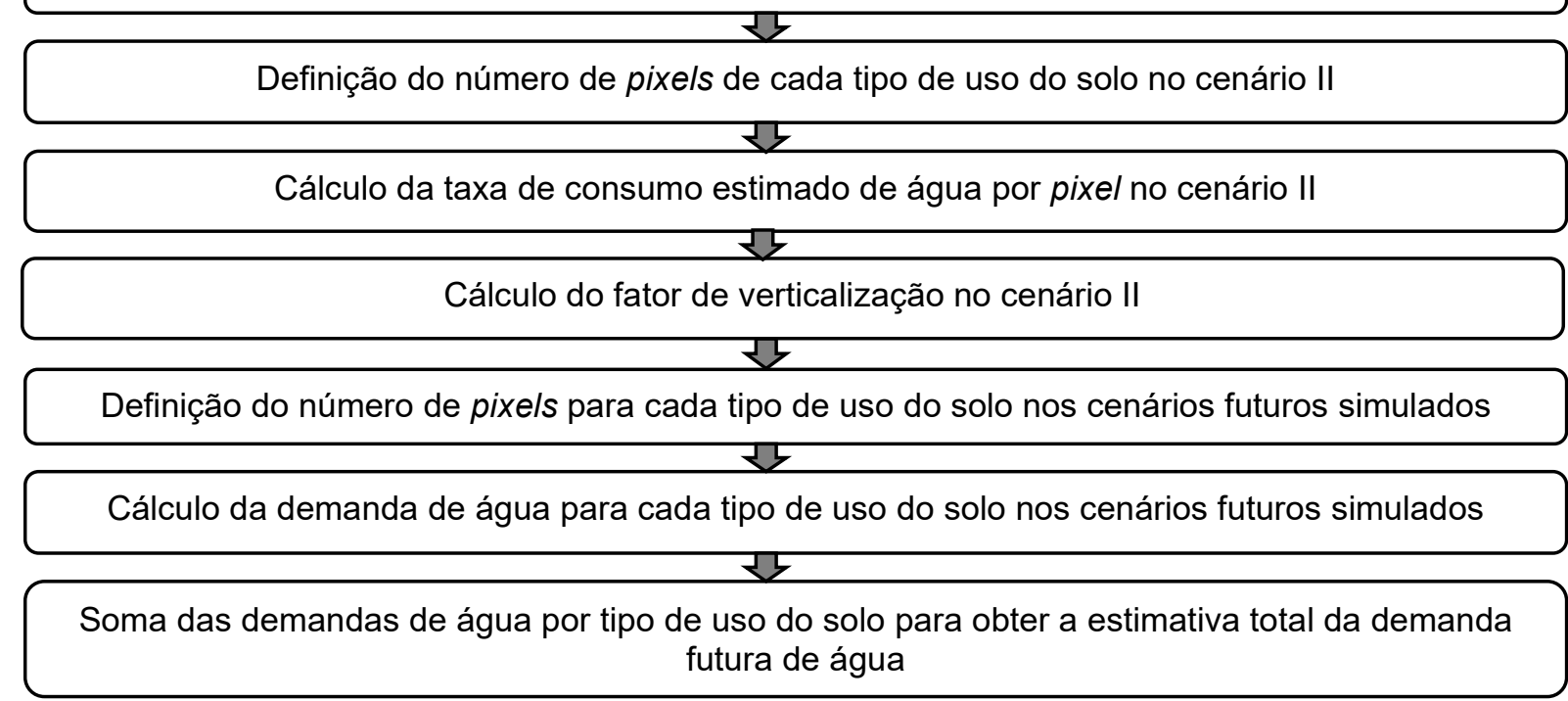

Fonte: elaborada pelo autor

Figura 6 - Síntese das etapas realizadas para estimar a demanda de água futura. 
e disponibilizados pela Cagepa. Essa comparação ocorreu apenas com o consumo normal estimado e o consumo medido, já que os valores medidos de 2018 disponibilizados pela Cagepa para a validação já não eram mais dados de um período de seca como o cenário estimado de consumo racional. Também não foi considerado nessa validação o uso com utilização de mecanismos poupadores, já que não havia confirmação de que no cenário real ocorria de fato a utilização de mecanismos poupadores.

\section{RESULTADOS E DISCUSSÃO}

Com relação às tendências de uso e à ocupação do solo da área de estudo, na Figura 3 percebe-se uma diminuição dos usos residencial, comercial e sem uso e uma tendência crescente de estabelecimentos prestadores de serviços na área estudada. Com relação à verticalização, percebe-se na Figura 4 que os lotes desocupados estão passando a ser ocupados por edifícios de múltiplos pavimentos, geralmente com uso misto predominante, desde dois pavimentos até edificações com mais de dez pavimentos, o que configura uma tendência de verticalização.

De acordo com os resultados dos índices V e JIU utilizados para testar a independência dos pares de variáveis e assim concluir a independência espacial dos mapas, nenhum par de variáveis apresentou correlação acima de $50 \%$ e, portanto, não precisou da exclusão de nenhuma variável ou da transformação de algum par de variáveis em uma única variável.

Na validação, o menor valor da similaridade fuzzy entre os mapas-diferença para os modelos de simulação de uso e ocupação do solo e de verticalização foram respectivamente, 0,49 e 0,57 para a validação utilizando função de decaimento exponencial em janela de tamanho 11x11. Novaes et al. (2011) afirmam que valores próximos a 0,40 indicam bom nível de compatibilidade. Trentin e Freitas (2010) também ressaltam que, tratando-se da dinâmica de áreas urbanas com morfologias dispersas, mesmo valores de similaridade relativamente baixos na escala de 0 a 1 podem ser considerados como aceitáveis.

Para a validação utilizando função de decaimento constante em janelas múltiplas, analisaram-se os valores das janelas de $3 \times 3$ e $5 \times 5$ pixels. O modelo de simulação de uso e ocupação do solo obteve valores de 0,48 (para a janela $3 \times 3$ ) e 0,50 (para a janela $5 \times 5$ ), enquanto o modelo de simulação da verticalização obteve valores de 0,56 (para a janela $3 \times 3$ ) e 0,58 (para a janela $5 \times 5$ ). De modo prático, índices de similaridade fuzzy adaptados com valores oscilando entre 0,45 e 0,50 para janelas com tamanhos de 3x3 a 5x5 têm denotado concordâncias aceitáveis (ALMEIDA et al., 2008; SOARES-FILHO; RODRIGUES; FOLLADOR, 2013). Adicionalmente, realizou-se um mapa de diferença entre o 2018 real e o 2018 simulado (Tabelas 4 e 5), pode-se observar, por meio do cálculo dos erros absoluto e relativo, que a quantidade de pixels dos dois mapas é bastante similar para os mapas tanto de uso e ocupação do solo como de verticalização.

O meio urbano é extremamente dinâmico e as relações de vizinhança entre os pixels passam a ser bem variadas mesmo de um ano para outro (comércios tornam-se serviços, residências tornam-se comércios etc.). Após um processo iterativo, um "presente simulado" foi comparado ao "presente real". Essa comparação se deu por meio de um mapa de diferença simples com uma quantificação dos pixels por classes de uso (Tabelas 4 e 5).

As Figuras 7 e 8 são uma quantificação em gráfico dos mapas obtidos e mostram que as mesmas tendências de uso e ocupação do solo e de verticalização
Tabela 4 - Calibração por comparação da quantidade de pixels: mapas de uso e ocupação do solo.

\begin{tabular}{l|c|c|c|c}
$\begin{array}{l}\text { Classes de } \\
\text { usos do } \\
\text { solo }\end{array}$ & 2018 real & $\begin{array}{c}2018 \\
\text { simulado }\end{array}$ & $\begin{array}{c}\text { Erro } \\
\text { absoluto }\end{array}$ & $\begin{array}{c}\text { Erro relativo } \\
(\%)\end{array}$ \\
\hline Residencial & 345.676 & 345.173 & 503 & 0,146 \\
\hline Comercial & 46.560 & 46.563 & 3 & 0,006 \\
\hline Misto & 58.622 & 58.626 & 4 & 0,007 \\
\hline Serviço & 187.810 & 187.810 & 0 & 0,000 \\
\hline Industrial & 3.213 & 3.217 & 4 & 0,124 \\
\hline Sem Uso & 130.861 & 131.353 & 492 & 0,376 \\
\hline
\end{tabular}

Fonte: elaborada pelo autor.

Tabela 5 - Calibração por comparação da quantidade de pixels: mapas de verticalização.

\begin{tabular}{l|c|c|c|c}
$\begin{array}{l}\text { Classes de } \\
\text { verticalização }\end{array}$ & $\begin{array}{c}2018 \\
\text { real } \\
\text { Desocupado }\end{array}$ & $\begin{array}{c}2018 \\
\text { simulado }\end{array}$ & $\begin{array}{c}\text { Erro } \\
\text { absoluto }\end{array}$ & $\begin{array}{c}\text { Erro } \\
\text { relativo (\%) }\end{array}$ \\
\hline 1 e 2 pavimentos & 536.797 & 536.843 & 46 & 0,001 \\
\hline 3 e 4 pavimentos & 67.060 & 66.954 & 106 & 0,009 \\
\hline 5 a 10 pavimentos & 20.257 & 20.277 & 20 & 0,099 \\
\hline $\begin{array}{l}\text { Acima de 10 } \\
\text { pavimentos }\end{array}$ & 14.242 & 14.280 & 38 & 0,267 \\
\hline
\end{tabular}

Fonte: elaborada pelo autor.

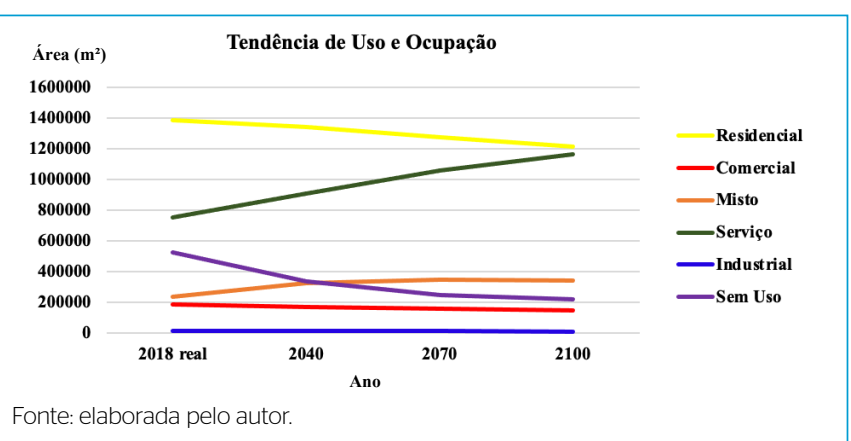

Figura 7 - Tendência de uso e ocupação do solo nos bairros Catolé e Sandra Cavalcante.

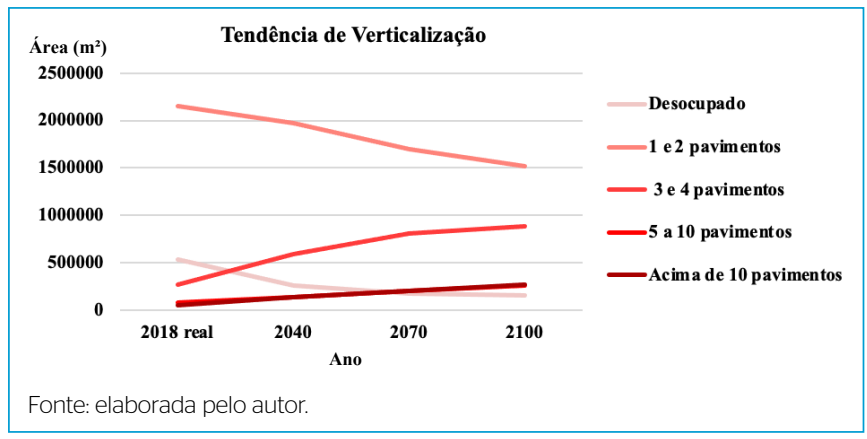

Figura 8 - Tendência de verticalização nos bairros Catolé e Sandra Cavalcante. 
que ocorreram entre os anos 2011 e 2018 continuaram ocorrendo e se confirmaram em longos períodos de tempo.

Com relação à tendência de verticalização (Figura 8) apresentada nos dois bairros, a simulação permitiu observar que as classes 1 e 2 (desocupado; 1 e 2 pavimentos) tendem a diminuir, dando espaço a áreas mais verticalizadas, por exemplo: clínicas médicas e estéticas e novos edifícios empresariais de caráter misto (com serviços comerciais e residências).

A Figura 9 apresenta os valores de consumo no cenário normal e no cenário racional, sem e com aplicação de mecanismos poupadores em ambos os cenários (com aplicação da taxa de redução de 10,42\%). Essa redução corresponde a uma diminuição de demanda de água SMP e CMP de 2.302.449 e 1.798.552 L/dia para o cenário de uso normal e uso racional, respectivamente.

Ante os cenários apresentados, percebe-se um aumento em torno de 300\% na demanda de água entre os anos 2018 e 2100 . Com isso, surge a preocupação sobre o atendimento a essas crescentes demandas de água, pois, de acordo com Meneses (2011), a cada ano os problemas de atendimento no sistema de abastecimento de Campina Grande vêm aumentando, haja vista o crescimento rápido das regiões e, principalmente, o sistema de abastecimento que não acompanha esse crescimento na mesma velocidade, tanto no aspecto da ampliação de suas unidades produtoras e distribuidoras como na melhoria dos processos operacionais e na minimização de perdas.

A validação dos dados de consumo de água foi uma etapa necessária pois, pelos dados obtidos, pode-se avaliar quão eficiente, do ponto de vista prático, encontra-se a metodologia aplicada. Na Tabela 6, é possível visualizar os dados de consumo estimado e medido (SMP) em L/dia para o mês de outubro de 2018, como também a diferença percentual entre eles.

De acordo com os dados apresentados na Tabela 6, ocorreu uma diferença de 10,59\% entre os valores de consumo de água estimado e medido (com mecanismos poupadores e sem mecanismos poupadores). Essa diferença pode ser explicada pelas aproximações das estimativas de consumo (número de usuários

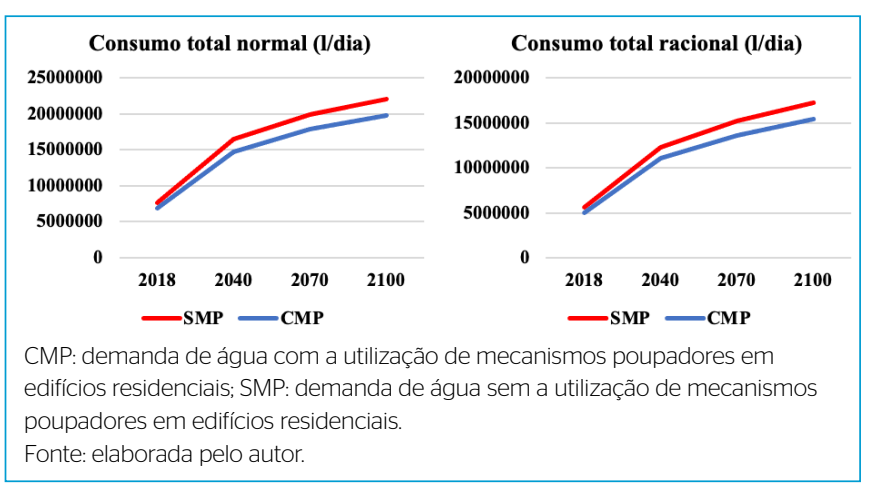

Figura 9 - Comparativo das demandas futuras simuladas.

Tabela 6 - Dados de consumo utilizados para validação.

\begin{tabular}{l|c|c|c} 
Cenários & Consumo estimado & Consumo medido & Diferença \\
\hline SMP & $7.645 .083,12$ & $8.454 .900,00$ & $10,59 \%$ \\
\hline
\end{tabular}

SMP: demanda de água sem a utilização de mecanismos poupadores em edifícios residenciais.

Fonte: elaborada pelo autor por imóvel etc.), além da utilização de um valor fixo estimativo de consumo per capita (micromedido) equivalente a 151,3 L/dia, correspondente ao ano de 2013 (dado mais recente disponível durante a pesquisa). Em contrapartida, o dado de consumo medido disponibilizado pela concessionária refere-se ao consumo micromedido em 2018, um ano em que a segurança hídrica promovida pelas águas advindas da transposição do rio São Francisco fez com que o consumo de água voltasse a patamares mais elevados.

\section{CONCLUSÕES}

Compreender o aumento do consumo de água e as tendências futuras é fundamental para um adequado planejamento dos serviços de infraestrutura urbana. Aliadas às previsões de mudanças de uso e ocupação do solo urbano, informações sobre a tendência de verticalização ou adensamento de uma área são fatores importantíssimos em planejamento de demandas hídricas, visto que os métodos tradicionais de estimativas de demandas futuras que consideram apenas as projeções de aumento populacional não são sensíveis às dinâmicas de uso e ocupação do solo que, muitas vezes, sobrecarregam os sistemas de abastecimento em áreas específicas de uma cidade.

Neste estudo, avaliou-se o crescimento da demanda de água em dois bairros de Campina Grande usando AC. Os mapas temáticos da demanda de água futura mostraram que esses bairros teriam um aumento significativo nessa demanda. Isso foi verificado com a comparação entre os mapas de consumo de água em 2018 e simulado de 2100, em que foi identificado um aumento de $300 \%$. Número preocupante, já que a cidade é abastecida por um único manancial que já correu risco de colapso. Em contrapartida, o número pode não ser tão alto como esse, já que uma mudança que ocorreu em sete anos foi extrapolada para horizontes de tempo de 22, 52 e 82 anos.

Esse período de sete anos de mudança sendo aplicado para horizontes de tempo mais longos pode ser justificado pela escassez de cenários de uso do solo urbano baseados em levantamentos de campo (ground truth), pois esses cenários não costumam ser prioridade para o planejamento municipal nas prefeituras. Mas também se deve levar em conta que, para modelos baseados em $\mathrm{AC}$, a dinamicidade do meio é muito mais imperativa do que a extensão (em termos temporais) do intervalo analisado.

Ante o potencial de simulação da dinamicidade do uso do solo urbano em modelos baseados em AC, mais especificamente do software utilizado, mudanças na legislação urbanística da cidade onde os bairros do estudo estão localizados ou novas pressões por déficit de água poderiam ser incluídas no modelo de simulação como variáveis dinâmicas, para obter novos resultados. O modelo de simulação é interativo.

Além disso, a validação da estimativa do consumo de água de 2018 apresentou resultados satisfatórios, com uma diferença entre o valor de cálculo, de acordo com a metodologia aplicada, e o valor medido pela Cagepa da ordem de $10,59 \%$.

\section{CONTRIBUIÇÕES DOS AUTORES}

Almino, L. M. O.: Conceituação, Coleta de Dados, Investigação, Metodologia, Processamento dos Dados, Escrita - Redação e Edição. Rufino, I. A. A.: Conceituação, Supervisão, Coordenação de Projeto, Obtenção e Financiamento, Investigação, Metodologia, Escrita — Redação e Edição. 


\section{REFERÊNCIAS}

AGÊNCIA EXECUTIVA DE GESTÃO DAS AGUAS DO ESTADO DA PARAÍBA (AESA). Monitoramento: volumes diários dos açudes. AESA, 2019. Disponível em: http://www.aesa.pb.gov.br/aesa-website/monitoramento/ volume-diario/. Acesso em: 8 set. 2021.

ALMEIDA, C. M. Modelagem da dinâmica espacial como uma ferramenta auxiliar ao planejamento: simulação de mudanças de uso da terra em áreas urbanas para as cidades de Bauru e Piracicaba (SP), Brasil. Tese (Doutorado) - Programa de Pós-Graduação em Sensoriamento Remoto, Instituto Nacional de Pesquisas Espaciais, São José dos Campos, 2003.

ALMEIDA, C. M.; CAMARA, G.; MONTEIRO, A. M. V. Geoinformação em urbanismo: cidade real x cidade virtual. São Paulo: Oficina de Textos, 2007.

ALMEIDA, C. M.; GLERIANI, J. M.; CASTEJON, E. F.; SOARES-FILHO, B. S. Using neural networks and cellular automata for modelling intra-urban land-use dynamics. International Journal of Geographical Information Science, v. 22, n. 9, p. 943-963, set. 2008. https://doi.org/10.1080/13658810701731168

ARAÚJO, E. L. Estimativa e análise do crescimento da demanda de água considerando cenários de uso e ocupação do solo. Dissertação (Mestrado) - Programa de Pós-Graduação em Engenharia Civil e Ambiental, Universidade Federal de Campina Grande, Campina Grande, 2012.

BARROS, M. B.; RUFINO, I. A. A.; MIRANDA, L. I. B. Mecanismos poupadores de água como suporte ao planejamento urbano. Revista Brasileira de Recursos Hidricos, v. 21, n. 1, p. 251-262, 2016. https://doi.org/10.21168/rbrh.v21n1.p251-262

BONHAM-CARTER, G. F. Geographic information systems for geoscientists: modelling with GIS. Ontário: Pergamon, 1994.

COMPANHIA DE AGUA E ESGOTOS DA PARAÍBA (CAGEPA). Relatório de Informações Cadastrais. Campina Grande: CAGEPA, 2018.

COMPANHIA DE SANEAMENTO BÁSICO DO ESTADO DE SÃO PAULO (SABESP). NTS 181: Dimensionamento do ramal predial de água, cavalete e hidrômetro - Primeira ligação. Norma Técnica. São Paulo: Diretoria de Tecnologia, Empreendimentos e Meio Ambiente, 2012.

DELANEZE, M. E. Modelagem dinâmica espacial aplicada na avaliação das mudanças da cobertura da terra no entorno de dutos utilizando autômatos celulares - estudo de caso duto orbel. Dissertação (Mestrado) - Instituto de Geociências e Ciências Exatas, Universidade Estadual Paulista "Júlio de Mesquita Filho", Rio Claro, 2011.

DIAS, L. T.; WALDE, D. H. G. Modelagem da dinâmica espacial do uso e ocupação do solo na Bacia Hidrográfica do Lago Paranoá - DF. Revista Brasileira de Cartografia, v. 65, n. 1, p. 77-94, 2013

FERRARI, F. B.; LAPOLLI, E. M. Uma proposta de avaliação da expansão urbana na ilha de Santa Catarina utilizando técnicas de sensoriamento remoto. In: SIMPOSIO LATINOAMERICANO DE PERCEPCIÓN REMOTA Y SISTEMAS DE INFORMACIÓN ESPACIAL, 9., 2000. Anais [...]. 2000.

FIGUERES, C. Urban water management in the Middle East and Central Asia. In: WORLD WATER CONGRESS OF IWRA, 12., 2005, Nova Délhi. Anais [...]. Nova Délhi, 2005

GALVÃO, C. O.; RÊGO, J. C.; RIBEIRO, M. M. R.; ALBUQUERQUE, J. P. T. Sustainability characterization and modelling of water supply management practices. Regional Management of Water Resources, n. 268, p. 81-88, 2001.
GARDNER, M. Mathematical games: the fantastic combinations of John Conway's new solitaire game "life". Scientific American, v. 223, p. 120-123, 1970

GONÇALVES, D. A.; SOUSA JUNIOR, W. C.; ALMEIDA, C. M. Modelagem de alteração de uso e cobertura da terra em uma porção do município de mineiros-go utilizando o método de pesos de evidência. In: SIMPÓSIO BRASILEIRO DE GEOGRAFIA FISICA APLICADA, 2007. Anais [...]. 2007. p. 13.

GONÇALVES, R. M.; CENTENO, T. M.; CANDEIAS, A. L. B. Cellular automata applied to tendency models in multi-temporal images. Revista Brasileira de Cartografia, v. 63, n. 2, p. 233-241, 2011

GONG, W:; YUAN, L.; FAN, W:; STOTT, P. Analysis and simulation of land use spatial pattern in Harbin prefecture based on trajectories and cellular automata-markov modelling. International Journal of Applied Earth Observation and Geoinformation, v. 34, p. 207-216, 2015. https://doi. org/10.1016/j.jag.2014.07.005

GUEDES, M. J. F.; RIBEIRO, M. M. R.; VIEIRA, Z. M. C. Alternativas de gerenciamento da demanda de água na escala de uma cidade. Revista Brasileira de Recursos Hidricos, Porto Alegre, v. 19, n. 2, p. 123-134, 2014. http:// dx.doi.org/10.21168/rbrh.v19n2.p123-134

HAGEN, A. Fuzzy set approach to assessing similarity of categorical maps, International Journal of Geographical Information Science, v. 17, n. 3, p. 235249, 2003. https://doi.org/10.1080/13658810210157822

INSTITUTO BRASILEIRO DE GEOGRAFIA E ESTATISTICA (IBGE). Censo demográfico. IBGE, 2010. Disponível em: https://www.ibge.gov.br/ estatisticas-novoportal/sociais/populacao/9662-censo-demografico-2010, html?=\&t=o-que-e. Acesso em: 28 nov. 2017.

INSTITUTO BRASILEIRO DE GEOGRAFIA E ESTATISTICA (IBGE). Cidades $e$ estados. IBGE, 2018a. Disponível em: https://www.ibge.gov.br/informacoes-porcidade-e-estado.html?t=destaques\&c=2504009. Acesso em: 28 nov. 2018.

INSTITUTO BRASILEIRO DE GEOGRAFIA E ESTATÍSTICA (IBGE). Relação Anual de Informações Sociais. IBGE. Disponível em: https://ces.ibge.gov.br. Acesso em: 20 set. 2018b.

JAT, M. K.; CHOUDHARY, M.; SAXENA, A. Urban growth assessment and prediction using RS, GIS and SLEUTH model for a heterogeneous urban fringe. The Egyptian Journal of Remote Sensing and Space Science, v. 20, n. 2. p. 223-241, 2017. http://dx.doi.org/10.1016/j.ejrs.2017.02.002

JOHANSSON, A.; BATTY, M.; HAYASHI, K.; AL BAR, O.; MARCOZZI, D.; MEMISH, Z. Crowd and environmental management during mass gatherings. Lancet Infectious Diseases, v. 12, n. 2, p. 150-156, 2012. https://doi.org/10.1016/S14733099(11)70287-0

MAEDA, E. E.; ALMEIDA, C. M.; XIMENES, A. C.; FORMAGGIO, A. R. SHIMABUKURO, Y. E. Dynamic modeling of forest conversion: Simulation of past and future scenarios of rural activities expansion in the fringes of the Xingu National Park, Brazilian Amazon. International Journal of Applied Earth Observation and Geoinformation, v. 13, n. 3, p. 435-446, 2011. https:// doi.org/10.1016/j.jag.2010.09.008

MALLESON, N.; BIRKIN, M. Towards victim-oriented crime modelling in a social science e-infrastructure. Philosophical Transactions of the Royal Society: Mathematical, Physical and Engineering Sciences, v. 369, n. 1949, p. 3353-3371, 2011. https://doi.org/10.1098/rsta.2011.0142 
MAS, J. F.; KOLB, M.; PAEGELOW, M.; OLMEDO, M. T. C.; HOUET, T. Inductive pattern-based land use/cover change models: a comparison of four software packages. Environmental Modelling and Software, v. 51, p. 94-111, 2014. https://doi.org/10.1016/j.envsoft.2013.09.010

MASSABKI, J. A. R.; PEIXOTO, A. S. P.; KAISER, I. M.; MANZATO, G. G. Modelagem dos padrões da expansão urbana da Região Metropolitana de São Paulo baseada em Autômatos Celulares. Revista Brasileira de Gestão Urbana, v. 9 , supl. 1, p. 361-371, 2017. https://doi.org/10.1590/2175-3369.009.SUPL1.AOO8

MATTHEWS, R.; GILBERT, N.; ROACH, A.; POLHILL, J.; GOTTS, N. Agentbased land-use models: a review of applications. Landscape Ecology, v. 22, n. 10, p. 1447-1459, 2007. https://doi.org/10.1007/s10980-007-9135-1

MEIRELLES, M. S. P.; CAMARA, G.; ALMEIDA, C. M. Geomática: modelos e aplicações ambientais. Brasília: Embrapa Informação Tecnológica, 2007.

MENESES, R. A. Diagnóstico operacional de sistemas de abastecimento de água: o caso de Campina Grande. Dissertação (Mestrado) - Programa de Pós-Graduação em Engenharia Civil e Ambiental, Universidade Federal de Campina Grande, Campina Grande, 2011.

NOVAES, M. R;; ALMEIDA, C. M.; RUDORFF, B. F. T:; AGUIAR, D. A. Cenários prognósticos baseados em modelagem dinâmica espacial para o manejo da colheita da cana-de-açúcar no estado de São Paulo. In: SIMPÓSIO BRASILEIRO DE SENSORIAMENTO REMOTO, 15., 2011. Anais [...]. 2011. p. 407-414

RÊGO, J. C.; ALBUQUERQUE, J. P. T.; RIBEIRO, M. M. R. Uma análise da crise de 1998-2000 no abastecimento de água de Campina Grande. In: SIMPÓSIO DE RECURSOS HÍDRICOS DO NORDESTE, 5., 2000. Anais [...]. Natal: ABRH, 2000

RÊGO, J. C.; GALVÃO, C. O.; ALBUQUERQUE, J. P. T. Considerações sobre a gestão dos recursos hídricos do açude Epitácio Pessoa-Boqueirão na bacia hidrográfica do rio Paraíba em cenário de vindouros anos secos. In: SIMPÓSIO DE RECURSOS HÍDRICOS DO NORDESTE, 11., 2012. Anais [...]. João Pessoa: ABRH, 2012.

RÊGO, J. C.; GALVÃO, C. O.; VIEIRA, Z. M. C. L.; RIBEIRO, M. M. R.; ALBUQUERQUE, J. P. T.; SOUZA, J. A. Atribuições e responsabilidades na gestão dos recursos hídricos - O caso do açude Epitácio Pessoa/Boqueirão no Cariri paraibano. In: SIMPOSIO BRASILEIRO DE RECURSOS HÍDRICOS, 20., 2013. Anais [...]. 2013. p. 1-8.
ROSSETTI, L. A. F. G.; ALMEIDA, C. M.; PINTO, S. A. F. Análise de mudanças no uso do solo urbano e rural com a aplicação de modelagem dinâmica espacial. In: SIMPÓSIO BRASILEIRO DE SENSORIAMENTO REMOTO, 16. 2013. Anais [...]. 2013. p. 6316-6323.

ROY, G. G.; SNICKARS, F. Citylife: a study of cellular automata in urban dynamics. In: FISHER, M. (org.). Spatial analytical perspectives on GIS. Londres: Taylor \& Francis, 1996. p. 213-218.

SAKIEH, Y.; AMIRI, B. J.; DANEKAR, A.; FEGHHI, J.; DEZHKAM, S. Simulating urban expansion and scenario prediction using a cellular automata urban growth model, SLEUTH, through a case study of Karaj City, Iran. Journal of Housing and the Built Environment, v. 30, n. 4, p. 591-611, 2015. https://doi. org/10.1007/s10901-014-9432-3

SISTEMA NACIONAL DE INFORMAÇÕES SOBRE SANEAMENTO (SNIS). Portal. Disponível em: http://www.snis.gov.br/. Acesso em: 15 set. 2018.

SOARES-FILHO, B. S.; CERQUEIRA, G. C.; PENNACHIN, C. L. Dinamica: a stochastic cellular automata model designed to simulate the landscape dynamics in an Amazonian colonization frontier. Ecological Modelling, v. 154, n. 3, p. 217-235, 2002. https://doi.org/10.1016/S0304-3800(02)00059-5

SOARES-FILHO, B. S.; RODRIGUES, H.; FOLLADOR, M. A hybrid analyticalheuristic method for calibrating land-use change models. Environmental Modelling and Software, v. 43, p. 80-87, 2013. https://doi.org/10.1016/j. envsoft.2013.01.010

SUAREZ, A. F.; SOARES-FILHO, B. S. Estudo da mudança de uso e cobertura do solo na bacia do Rio Formiga - MG. Revista Brasileira de Cartografia, v. 65, n. 3, p. 417-429, 2013.

TRENTIN, G. A expansão urbano-industrial do Município de Americana SP. Dissertação (Mestrado) - Instituto de Geociências e Ciências Exatas, Universidade Estadual Paulista "Júlio de Mesquita Filho", Rio Claro, 2008.

TRENTIN, G.; FREITAS, M. I. C. Modelagem dinâmica espacial urbana: modelo de autômato celular simulação de cenários para o município de Americana - SP. Revista Brasileira de Cartografia, v. 62, n. 1, p. 291-305, 2010.

TSUTYIA, M. T. Abastecimento de água. 3. ed. São Paulo: Departamento de Engenharia Hidráulica e Sanitária da Escola Politécnica da Universidade de São Paulo, 2006 